\title{
COMPARAÇ̃̃O ENTRE A META-HEURÍSTICA SIMULATED ANNEALING E A PROGRAMAÇÃO LINEAR INTEIRA NO AGENDAMENTO DA COLHEITA FLORESTAL COM RESTRIÇÕES DE ADJACÊNCIA
}

\author{
COMPARISON THE METAHEURISTIC SIMULATED ANNEALING AND INTEGER LINEAR \\ PROGRAMMING FOR SOLVING THE FOREST HARVEST SCHEDULING WITH ADJACENCY \\ CONSTRAINTS
}

\author{
Lucas Rezende Gomide ${ }^{1}$ Júlio Eduardo Arce ${ }^{2}$ Arinei Carlos Lindbeck da Silva ${ }^{3}$ \\ RESUMO
}

\begin{abstract}
Os impactos gerados na paisagem após a colheita florestal em reflorestamentos são visíveis, porém, o corte raso é um processo necessário para garantir uma produção sustentada e introduzir novas tecnologias. Uma alternativa de controle é utilizar restrições de adjacência nos modelos matemáticos. Assim, o objetivo do estudo foi avaliar a capacidade da meta-heurística SA na resolução de modelos matemáticos com restrições de adjacência do tipo URM, e observar sua ação com o aumento da complexidade do problema. $O$ estudo foi conduzido em um projeto florestal contendo 52 talhões, sendo criados 8 cenários, onde o modelo I de Johnson e Scheurmann (1977) foi usado como referência. A restrição de adjacência do tipo URM foi usada para controlar o corte de talhões adjacentes. Os modelos foram resolvidos pela PLI e meta-heurística SA, no qual foi processada 100 vezes/cenário. Os resultados mostraram que o cenário 8 consumiu 137.530 segundos via PLI, gastando um tempo de 2.023,09 vezes a mais que o tempo médio de processamento da metaheurística SA (67,98 segundos). As melhores soluções ficaram 4,71 \% (cenário 1) a 11,40 \% (cenário 8) distante do ótimo (PLI). A meta-heurística SA é capaz de resolver o problema florestal, atendendo às metas na maioria das vezes. $\mathrm{O}$ aumento da complexidade produz um maior desvio em relação ao ótimo. Concluise que a meta-heurística SA não deve ser processada uma única vez, pois há riscos de se obter soluções inferiores, caso seja feita, deve-se aumentar o tempo de parada.
\end{abstract}

Palavras-chave: inteligência artificial; programação linear inteira; colheita florestal.

\begin{abstract}
The impacts on the landscape after forest harvesting in reforestation are visible, but the cutting is a necessary process to ensure a sustained yield and introduce new technologies. An alternative of control is to use the adjacency constraints in the mathematical models. Thus, the aim of the study was to assess the ability of the metaheuristic SA to solve mathematical models with adjacency constraints type URM, and to check its action with the increasing of the problem complexity. The study was conducted in a forest project containing 52 stands, and created 8 scenarios, where the Johnson and Scheurmann (1977) model I was used as reference. The adjacency constraint type URM was used to control the cutting of adjacent stands. The models were solved by the ILP and metaheuristic SA, which was sued 100 times per scenario. The results showed that the scenario 8 has consumed 137,530 seconds via PLI, which represented 2,023.09 times more than the average time processing of the SA metaheuristic (67.98 seconds). The best solutions were $4.71 \%$ (scenario 1) to $11.40 \%$ (scenario 8) far from the optimal (ILP). The metaheuristic SA is capable to solve the forest problem, meeting the targets in the most cases. The increasing of complexity produced a higher deviation from the optimal. Concludes that the metaheuristic SA should not be processed a single time, because there are hazards in obtain inferior solutions, but doing it is recommended to increase the stop criterion.
\end{abstract}

Keywords: artificial intelligence; integer linear programming; forest harvest.

1. Engenheiro Florestal, Dr., Professor Adjunto do Departamento de Ciências Florestais, Universidade Federal de Lavras, Caixa Postal 3037, CEP 37200-000, Lavras (MG). lucasgomide@dcf.ufla.br

2. Engenheiro Florestal, Dr., Professor Adjunto do Departamento de Ciências Florestais, Universidade Federal do Paraná, CEP 80210-170, Curitiba (PR). jarce@ufpr.br

3. Licenciado em Matemática, Dr., Professor Associado do Departamento de Engenharia da Produção, Universidade Federal do Paraná, CEP 80210-170, Curitiba (PR). arineicls@gmail.com

Recebido para publicação em 11/02/2011 e aceito em 10/04/2012 


\section{INTRODUÇÃO}

Os modelos de programação linear (PL) aplicados ao manejo florestal, nas últimas décadas, constituíam-se, quase que exclusivamente, de restrições envolvendo objetivos volumétricos, da área ou região a ser manejada, onde a meta principal era regular a produção da floresta e maximizar o valor presente líquido ao longo de um dado horizonte de planejamento. Nessa etapa, o uso de variáveis contínuas nos modelos matemáticos era uma tendência. Entretanto, uma nova categoria de restrições técnicas de produção passou a exigir o uso de variáveis inteiras, para atender aos objetivos vinculados ao planejamento florestal. Essa mudança no tipo de variáveis de decisão foi necessária devido a questões espaciais retratadas, sendo expressa pela interação entre as unidades de produção (MURRAY e SNYDER, 2000). Assim, aspectos ambientais passaram a definir restrições ao sistema de produção, com o propósito de controlar ou mitigar os possíveis distúrbios gerados após a colheita florestal, como exemplos, tem-se o caso das restrições de adjacência descritas nos trabalhos de Weintraub et al. (1994), Brumelle et al. (1998), Borges e Hoganson (1999), Weintraub et al. (2000), Boston e Benttinger (2001), Murray et al. (2004), Goycooela et al. (2005) e Zhu et al. (2007). No Brasil, podem-se citar os trabalhos de Alonso (2003), Castro (2007) e Gomide et al. (2010). Os objetivos espaciais ainda podem envolver tamanho, forma e ligação entre unidades de manejo, influenciando nas operações de colheita florestal.

Os impactos gerados na paisagem após a colheita florestal em reflorestamentos são visíveis, podendo conter extensas áreas com exposição do solo, quebra da continuidade e retalhamento da paisagem, aumento de riscos causados por vendavais, dentre outros. Entretanto, França, Alemanha, Escandinávia e alguns países da Europa central têm limitado a área de corte raso, considerando conceitos de sustentabilidade e planejamento da paisagem (BELL e APOSTOL, 2008). O planejamento dessa atividade é essencial, tendo em vista a duração de um empreendimento florestal e seus impactos sobre a paisagem. Por outro lado, o corte raso é um processo necessário para garantir uma produção sustentada, determinando ainda o momento de fechamento da rotação cultural. Além disso, essa etapa permite a introdução de novas tecnologias silviculturais e alterações no regime de manejo seguido.

Historicamente, a resolução de problemas florestais envolvendo restrições de adjacência teve início na década de 70. Foi então formulada a restrição do tipo URM (unit restriction model), a qual, posteriormente, sofreu alterações conceituais gerando uma nova categoria conhecida como ARM (area restriction model). A diferença entre elas está na flexibilidade em aceitar ou não o corte de unidades de manejo vizinhas (ARM), considerando um determinado critério específico, contudo, ambas as abordagens se assemelham na necessidade do uso de variáveis inteiras (GOMIDE et al., 2010).

Silva et al. (2003) afirmam que os resultados operacionais provenientes de processos de arredondamentos de soluções obtida via PL (programação linear) não devem ser adotadas. A explicação foi mostrada em seu estudo, indicando que certas restrições e objetivos não puderam ser atendidos após o arredondamento. Dessa forma, a inclusão de variáveis inteiras muda consideravelmente a natureza da região factível, a qual deixa de ser contínua e passa a ser limitada por um número finito de pontos, e assim não há nenhuma garantia de que a solução ótima esteja no ponto extremo do plano, conforme observado na PL.

Hooker (2008) considera que os métodos para a resolução de problemas envolvendo a programação linear inteira (PLI) não são robustos quando comparados com a PL, onde a diferença é proporcionada pela categoria em que se enquadra esse tipo de problema (NP-Hard). Alguns problemas podem consumir um elevado tempo computacional para a resolução, mesmo considerando os melhores equipamentos disponíveis atualmente. Por outro lado, nas décadas de 80 e 90, inúmeras heurísticas e meta-heurísticas de busca foram desenvolvidas para a obtenção de soluções em tempo computacional compatível, como foi o caso das técnicas conhecidas como algoritmo genético, busca tabu, simulated annealing (SA), dentre outros (HERRMAN, 2006). Essa classe de métodos inteligentes de busca só foi desenvolvida após constatar a ineficiência dos métodos tradicionais em relação ao tempo computacional (BASKENT, 2001).

Segundo Glover e Kochenberger (2003), as meta-heurísticas são métodos de resolução que orquestram uma interação entre procedimentos de melhoria local e apuradas estratégias, com a finalidade de escapar de ótimos locais. A lógica na meta-heurística SA foi inspirada na têmpera (annealing), na qual se descreve um processo de 
aquecimento e posterior resfriamento de um metal a partir de reduções gradativas de temperatura, com o propósito de evitar a formação de defeitos no metal. O objetivo central do método é minimizar a energia do sistema durante o processo de redução de temperatura (LAARHOVEN e AARTS, 1987). Suman e Kumar (2006) comentam que essa meta-heurística é um método compacto e robusto, apresentando excelentes soluções para problemas de otimização, em reduzido tempo computacional.

Sendo assim, os objetivos do estudo foram: analisar a capacidade da meta-heurística SA na resolução de modelos matemáticos envolvendo restrições espaciais de adjacência do tipo URM, em problemas de agendamento da colheita florestal; comparar os resultados obtidos com o ótimo gerado pela programação linear inteira; e, identificar o comportamento da meta-heurística SA no aumento da complexidade do problema.

\section{MATERIAL E MÉTODOS}

\section{Área de estudo}

$\mathrm{O}$ estudo foi desenvolvido em 5 fazendas pertencentes à empresa VCP - Votorantim Celulose e Papel (atualmente FIBRIA), sendo todas localizadas no município de Caçapava-SP. O clima da região é denominado pela classificação de Köppen como Cwa, com verões chuvosos e quentes, e invernos secos. A área total efetiva de plantio compreendeu 1.490,7 ha, distribuídos em reflorestamentos com espécies de eucalipto e algumas variações de clones da empresa. O projeto designado para o estudo contabilizou uma malha de 52 unidades de manejo florestal ou talhões.

\section{O problema teste}

O planejamento de suprimento de madeira é normalmente parte integrante do manejo flores- tal, tendo como objetivos definir a agenda de corte das unidades e auxiliar na regulação da floresta (HOTVEDT, 1983). O seu agendamento representa um ponto estratégico para a empresa florestal, onde se dimensionam uma série de atividades de logísticas e procedimentos técnicos na colheita, em virtude da sequência de talhões agendados para a colheita.

Assim, o problema teste foi caracterizado pela necessidade de maximizar o valor presente líquido (VPL), analogamente ao encontrado em uma série de trabalhos na literatura, e que tiveram o modelo I de Johnson e Scheurmann (1977) como referência. Dessa maneira, o uso do indicador econômico VPL foi aplicado apenas para atualizar as receitas líquidas geradas por cada alternativa de manejo, não sendo aplicado como método de comparação entre alternativas de manejo na sua forma pura de análise. Além disso, foram introduzidas restrições de adjacência.

As restrições de adjacência do tipo URM impedem o corte de quaisquer talhões adjacentes do projeto para o mesmo período de tempo (Figura 1). Esse tipo de restrição é considerado muito rígido, por não contemplar a área dos talhões envolvidos para o corte, quando comparado com outras opções de restrições de adjacência. Na construção dessas restrições foi necessário gerar uma matriz de adjacência entre talhões, onde sua definição seguiu um processo criterioso, pois a área de estudo é composta por estradas, rodovias, linhas de transmissão de energia elétrica e gasodutos.

O problema teste foi dividido em 8 cenários, de acordo com o período de tempo do horizonte de planejamento (HP), o qual variou de 7 a 14 anos, conforme apresentado na Tabela 1. As restrições de integralidade foram usadas para garantir a seleção de apenas uma prescrição de manejo por talhão. Todos os cenários tiveram uma demanda constante anual de madeira de $69.000 \mathrm{~m}^{3}$, com variação de \pm

(Solução Infactível)
\begin{tabular}{|c|c|c|}
\hline Talhão 1 & Talhão 2 & Talhão 3 \\
\hline Talhão 4 & Talhão 5 & Talhão 6 \\
\hline Talhão 7 & Talhão 8 & Talhão 9 \\
\hline
\end{tabular}

FIGURA 1: Exemplo do agendamento da colheita florestal considerando o uso da restrição URM em duas situações de resposta (factível/infactível) no tempo t.

FIGURE 1: Example of forest harvest scheduling considering the use of URM constraint in two types of solutions (feasible/infeasible) at time t. 
TABELA 1: Caracterização dos cenários utilizados na comparação entre a meta-heurística SA e a programação linear inteira.

TABLE 1: Sceneries characterization applied to compare the metaheuristic simulated annealing and the integer linear programming.

\begin{tabular}{cccccc}
\hline \multirow{2}{*}{ Cenário } & \multirow{2}{*}{$\begin{array}{c}\text { Horizonte de planejamento } \\
\text { (anos) }\end{array}$} & $\begin{array}{c}\text { Alternativas de } \\
\text { manejo }\end{array}$ & \multicolumn{3}{c}{ Restrições } \\
\cline { 4 - 6 } 1 & 7 & 254 & Volume & Integralidade & Urm \\
2 & 8 & 323 & $\mathrm{x}$ & $\mathrm{x}$ & $\mathrm{x}$ \\
3 & 9 & 362 & $\mathrm{x}$ & $\mathrm{x}$ & $\mathrm{x}$ \\
4 & 10 & 384 & $\mathrm{x}$ & $\mathrm{x}$ & $\mathrm{x}$ \\
5 & 11 & 444 & $\mathrm{x}$ & $\mathrm{x}$ & $\mathrm{x}$ \\
6 & 12 & 571 & $\mathrm{x}$ & $\mathrm{x}$ & $\mathrm{x}$ \\
7 & 13 & 758 & $\mathrm{x}$ & $\mathrm{x}$ & $\mathrm{x}$ \\
8 & 14 & 939 & $\mathrm{x}$ & $\mathrm{x}$ & $\mathrm{x}$ \\
\hline
\end{tabular}

$42 \%$ de sua meta. A definição dos limites de produção volumétrica foi proposta em virtude de testes preliminares, nos quais uma porcentagem mais conservadora de desvio gerou sempre soluções infactíveis, no modelo de programação linear inteira, inviabilizando a comparação com a meta-heurística SA. A taxa de juros foi fixada em $10 \%$ ao ano em todos os cenários simulados, onde os custos de manutenção da floresta, de colheita e transporte foram informados pela empresa, bem como o preço de venda da madeira fixado em $\mathrm{R} \$ 76,00 / \mathrm{m}^{3}$.

A idade mínima e máxima permitida de corte foram, respectivamente, 5 e 7 anos, para a construção das alternativas de manejo. Ainda foi permitido deixar o talhão ocioso, ou seja, sem definir nenhuma opção de manejo para o mesmo. O sistema silvicultural considerado foi o alto fuste, onde ao cortar um talhão na sua totalidade, o mesmo material genético seria plantado na sequência, a ciclos contínuos. As projeções de crescimento volumétrico de madeira na floresta foram seguidas segundo dados da própria empresa.

Logo, o modelo de programação linear inteira utilizou uma função objetivo (1), com a finalidade de maximizar o valor presente líquido (VPL) em reais, sendo ainda composta pela restrição (2) de integridade de uso de uma única alternativa de manejo por talhão; (3) e (4) limites mínimos e máximos de produção volumétrica; (5) relacionada à restrição de adjacência do tipo URM e (6) para o uso de variáveis binárias. Dessa forma, o modelo de programação matemática empregado foi:

$$
\text { Função Objetivo: Maximizar } \quad \mathrm{VPL}=\sum_{\mathrm{i}=1 \mathrm{j}=1}^{\mathrm{M}} \underset{\mathrm{ij}}{\mathrm{P}} \mathrm{c}_{\mathrm{ij}} \mathrm{x}_{\mathrm{ij}}
$$

Sujeito a:

$$
\begin{array}{ll}
\sum_{\mathrm{j}=1}^{\mathrm{P}} \mathrm{X}_{\mathrm{ij}} \leq 1, & \forall_{\mathrm{j}}, \forall_{\mathrm{i}=1,2 \ldots \mathrm{M}} \\
\sum_{\mathrm{i}=1}^{\mathrm{M}} \sum_{\mathrm{j}=1}^{\mathrm{P}} \mathrm{V}_{\mathrm{ij}} \mathrm{X}_{\mathrm{ijT}} \geq \mathrm{L}_{\text {inferiot },} & \nabla_{\mathrm{i}}, \nabla_{\mathrm{j}}, \forall_{\mathrm{T}=1,2, \ldots \mathrm{HP}}
\end{array}
$$$$
\sum_{\mathrm{i}=1}^{\mathrm{M}} \sum_{\mathrm{j}=1}^{\mathrm{P}} \mathrm{V}_{\mathrm{ij}} \mathrm{x}_{\mathrm{ijT}} \leq \mathrm{L}_{\text {superior }}, \quad \forall_{\mathrm{i}}, \forall_{\mathrm{j}}, \forall_{\mathrm{T}=1,2, \ldots \mathrm{HP}}
$$

$$
\begin{array}{cc}
\sum_{\substack{\mathrm{i}=1 \\
\mathrm{i} \neq \mathrm{k}}}^{\mathrm{M}} \mathrm{a}_{\mathrm{ik}} \mathrm{x}_{\mathrm{ij} \mathrm{T}}+\sum_{\mathrm{i}=1 \mathrm{i}}^{\mathrm{M}} \cdot \mathrm{a}_{\mathrm{ik}} \cdot \mathrm{x}_{\mathrm{ij} \mathrm{T}} \leq \sum_{\mathrm{i}=1}^{\mathrm{M}} \mathrm{a}_{\mathrm{ik}}, \forall_{\mathrm{i}}, \forall_{k}, \forall_{\mathrm{j}}, \forall_{\mathrm{T}=1,2, \ldots \mathrm{HP}}(5) \\
\mathrm{x}_{\mathrm{ij}} \in\{0,1\} & \forall_{\mathrm{i}}, \forall_{\mathrm{j}}
\end{array}
$$

Em que: M: número total de talhões manejados; $\mathrm{P}$ : número total de alternativas de manejo; $\mathrm{x}_{\mathrm{ij}}$ : variável binária $(0 / 1)$ de decisão do modelo, referente ao talhão $i$ e alternativa de manejo $j$; $\mathrm{c}_{\mathrm{ij}}$ : valor presente líquido pertencente ao talhão $i$ segundo a alternativa de manejo $j$; T: período total de tempo de planejamento; $\mathrm{v}_{\mathrm{ij}}$ : volume total produzido no talhão $i$ pela alternativa de manejo $j$; $\mathrm{k}$ - talhão número; $a_{i k}$ - matriz binária $\{0,1\}$ de adjacência entre 
talhões, onde $i=k$; T: período de tempo; $\mathrm{L}_{\text {inferior }}$ : limite volumétrico mínimo de madeira permitido em $\mathrm{m}^{3} ; \mathrm{L}_{\text {superior: }}$ : limite volumétrico máximo de madeira permitido $\mathrm{em}^{3}$;

\section{Métodos de resolução \\ Programação linear inteira (PLI)}

O problema de agendamento da colheita florestal requer o uso de variáveis binárias devido à necessidade de colher totalmente um talhão, ao invés de fazê-lo parcialmente, e ainda respeitar o uso de restrições espaciais. Portanto, foi utilizada a PLI por meio do algoritmo branch-and-bound para a obtenção das soluções. Esse procedimento foi adotado como testemunha, uma vez que a PLI garante o ótimo.

\section{Meta-heurística simulated annealing}

No processo de programação da meta-heurística, adotou-se uma codificação binária $\{0,1\}$ para popular a string, ou seja, preencher os elementos de uma matriz de dimensão 1 x N. Esse código binário é um código de simples implementação e sua escolha foi em virtude da natureza do problema teste, sendo caracterizado por apresentar variáveis de decisão binária. Dessa maneira, a codificação da string presente na SA seguiu o esquema apresentado pela Figura 2. O termo $U$ representa cada talhão pertencente ao projeto florestal, onde o mesmo foi subdividido conforme o número de anos de avaliação do projeto (horizonte de planejamento).

A função de avaliação (energia) utilizada na meta-heurística levou em consideração a introdução de penalidades no modelo. Segundo Pezeshk e Camp (2002), o resultado da função ob- jetivo penalizada representa, de forma quantitativa, a extensão da violação da restrição e promove uma medida relativa da qualidade de cada solução mensurada.

A formulação matemática empregada na meta-heurística SA seguiu a mesma lógica descrita anteriormente, porém, foi introduzida na função objetivo (1) uma penalidade, transformando-a na função objetivo (7). O método de penalidades foi empregado para guiar nas buscas de soluções e contribuir para uma boa convergência da resposta. A partir de testes iniciais foram definidas as seguintes penalidades: $\mathrm{R} \$ 500,00 / \mathrm{m}^{3}$ de madeira fora da meta volumétrica (VOL), R $\$ 1.000 .000,00 /$ corte de talhões adjacentes, no mesmo período de tempo (URM). A formulação seguiu a seguinte estrutura:

$$
\begin{gathered}
\text { Max VPL }=\sum_{\mathrm{i}=1}^{M} \sum_{j=1}^{\mathrm{P}} \mathrm{c}_{\mathrm{ij}} \mathrm{x}_{\mathrm{ij}} \text { - penalidade (VOL) }{ }_{\mathrm{k}=1}^{\mathrm{T}} \mathrm{D}_{\mathrm{k}} \\
\text { - penalidade (URM) } \sum_{\mathrm{k}=1}^{\mathrm{T}} \mathrm{A}_{\mathrm{k}}
\end{gathered}
$$

Em que: Penalidade (VOL): penalidade em $\mathrm{R} \$ / \mathrm{m}^{3}$ de madeira fora do intervalo de produção definido; Penalidade (URM): penalidade em R $\$$ / número de ocorrências de corte de talhões vizinhos; D: Valor absoluto do desvio volumétrico de madeira $\left(\mathrm{m}^{3}\right)$; A: Número de ocorrências de corte de talhões vizinhos; $\mathrm{k}$ : período anual de tempo dentro do horizonte de planejamento; $\mathrm{M}, \mathrm{P}, \mathrm{x}_{\mathrm{ij}}, \mathrm{c}_{\mathrm{ij}} \mathrm{e}$ $\mathrm{T}$ : definidos anteriormente.

Os parâmetros iniciais utilizados na meta-heurística SA foram:

a) Temperatura inicial: 4.091 (após a realização de testes preliminares);

\section{STRING}
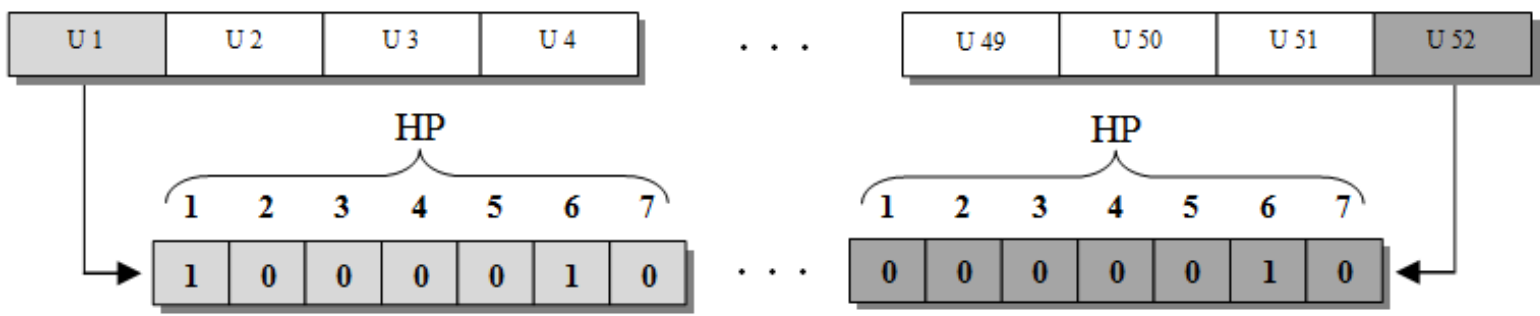

FIGURA 2: Exemplificação da codificação binária empregada na construção da meta-heurística simulated annealing considerando um horizonte de planejamento de 7 anos e 52 talhões.

FIGURE 2: Binary code exemplification applied to build the metaheuristic Simulated Annealing considering the horizon planning of 7 years and 52 stands. 
b) Função de resfriamento: $T_{i}=\{[(i-1)$ melhores soluções, considerando 60 iterações; $\left.\left.\mathrm{T}_{\mathrm{i}-1}+\mathrm{T}_{0}\right] \div[\mathrm{i}(1+\log (\mathrm{i}))]\right\}$

Onde:

A meta-heurística SA foi programada con-

$\mathrm{T}_{\mathrm{i}}$ - Temperatura do sistema na iteração $\mathrm{i}$;

$\mathrm{T}_{\mathrm{i}-1}$ - Temperatura do sistema na iteração i-1;

$\mathrm{T}_{0}$ - Temperatura inicial do sistema;

log - Logaritmo na base 10;

i - Iteração atual do processo.

c) Critério de parada: 30.000 iterações;

d) Distância de busca local: refere-se ao grau de perturbação na solução pesquisada, sendo uma mudança de $10 \%$ nas Us presentes na string a cada iteração;

e) Estratégia de programação da meta-heurística SA: explorar mais a vizinhança de busca das forme encontrado no trabalho de Kirkpatrick et al. (1983), iniciando com a definição de uma solução gerada randomicamente, porém, uma estratégia foi desenvolvida para melhorar as buscas e explorar mais a vizinhança das melhores soluções, conforme fluxograma da Figura 3.

\section{Implementação computacional e processamento}

A implementação da meta-heurística SA foi desenvolvida utilizando a linguagem de programação Visual Basic ${ }^{\circledR}$ versão 6.0 Enterprise da Microsoft ${ }^{\mathbb{B}}$, licenciada para o PPGEF (Programa de Pós-Graduação em Engenharia Florestal) da UFPR,

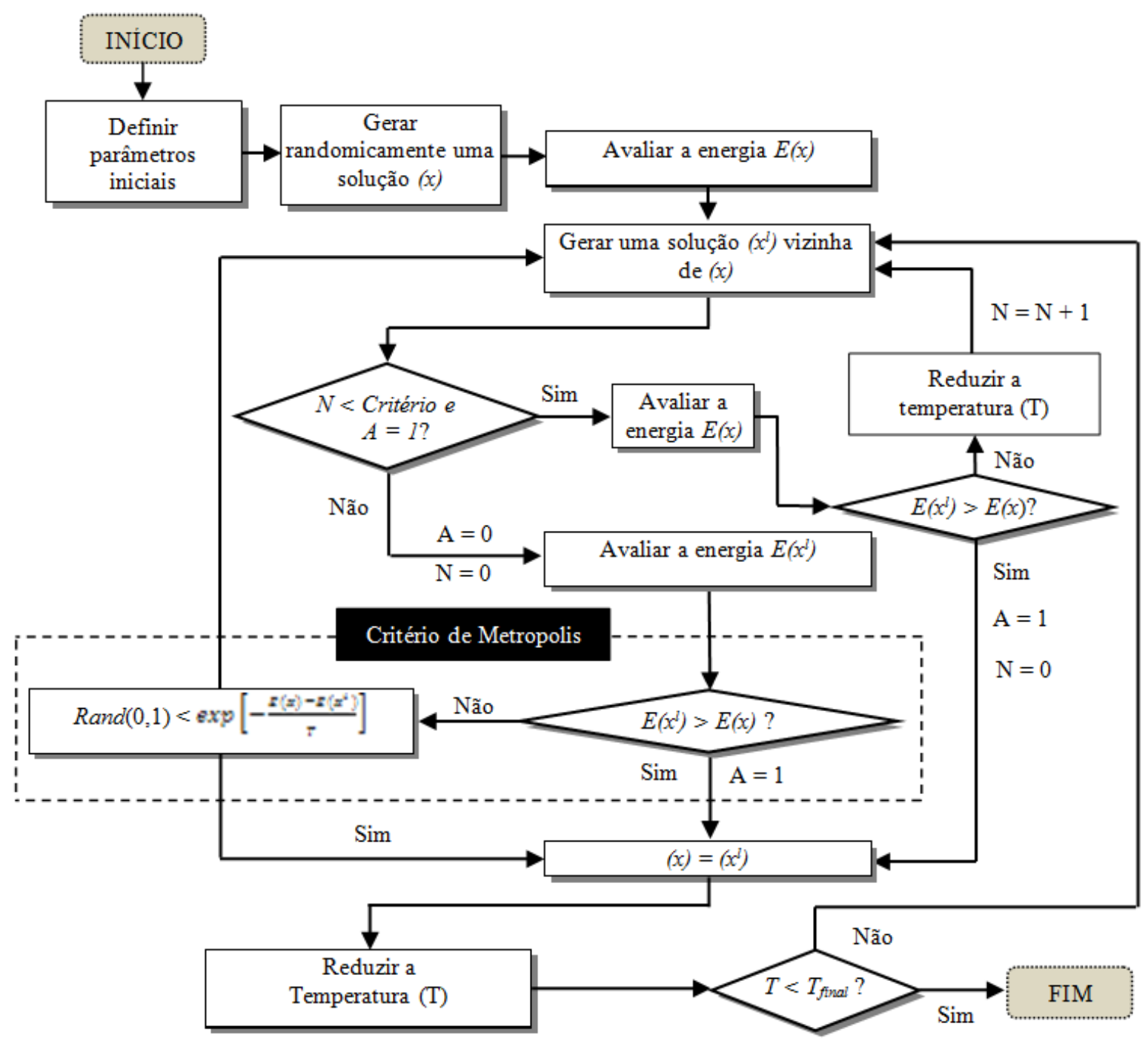

FIGURA 3: Estrutura da meta-heurística SA utilizando a estratégia de explorar mais a vizinhança de busca das melhores soluções.

FIGURE 3: The structure of metaheuristic SA using the strategy to explore more the neighborhood in searching for the best solutions. 
criando-se então uma ferramenta de cálculo. Os modelos matemáticos de PLI também foram escritos por esta ferramenta, porém, o seu processamento foi realizado através do software LINGO 9.0 (Language for Interactive General Optimizer). O processamento foi executado em um computador com processador Intel Xeon ${ }^{\circledR} 2,0 \mathrm{GHz}$ e $8 \mathrm{~Gb}$ de RAM, sendo executadas 100 repetições/cenário pela meta-heurística SA. As variáveis obtidas foram os valores de tempo de processamento, VPL, valores volumétricos anuais e capacidade da meta-heurística em atender à restrição URM, em cada processamento. Os dados foram analisados utilizando a estatística descritiva, mesma lógica empregada por Rodrigues et al. (2004).

\section{RESULTADOS E DISCUSSÃO}

Os modelos matemáticos que retratam objetivos espaciais apresentam um número elevado de restrições, pois, geralmente, envolvem análise combinatória, e por isso, quanto maior o problema, maior é esse número. A restrição de adjacência do tipo URM se enquadra nesse grupo de problema, onde a construção do modelo de PLI força rastrear todas as combinações espaciais existentes entre os talhões, para assegurar que talhões vizinhos não possam ser colhidos no mesmo tempo $t$ do horizonte de planejamento. No problema teste, essa afirmativa foi confirmada e o número total de restrições teve um aumento de $221,2 \%$ entre os cenários $1 \mathrm{e}$ 8 (Tabela 2). O maior percentual de restrições para os 8 cenários pertencia às restrições de adjacência do tipo URM, chegando a um valor de $85,53 \%$ do total de restrições no cenário com maior horizonte de planejamento, 14 anos (cenário 8), sendo observado ainda, que as restrições de integridade não sofreram mudanças em seu número. Por outro lado, a restri-
TABELA 2: Número de restrições presentes nos modelos de PLI para os 8 cenários.

TABLE 2: Number of constraints in the ILP for the 8 sceneries.

\begin{tabular}{cccccc}
\hline \multirow{2}{*}{ Cenários } & \multirow{5}{*}{ Hp } & \multicolumn{4}{c}{ Restrições } \\
\cline { 3 - 6 } & & Volume & Integridade & Urm & Total \\
\hline 1 & 7 & 14 & 52 & 184 & 250 \\
2 & 8 & 16 & 52 & 221 & 289 \\
3 & 9 & 18 & 52 & 258 & 328 \\
4 & 10 & 20 & 52 & 293 & 365 \\
5 & 11 & 22 & 52 & 338 & 412 \\
6 & 12 & 24 & 52 & 383 & 459 \\
7 & 13 & 26 & 52 & 428 & 506 \\
8 & 14 & 28 & 52 & 473 & 553 \\
\hline
\end{tabular}

ção volumétrica obteve um aumento de 2 restrições a cada alteração no HP, o qual se refere ao limite inferior e superior de produção.

O aumento na complexidade dos cenários promoveu um maior consumo de tempo computacional, para a obtenção das soluções, tanto via PLI como para a meta-heurística SA. O incremento no tempo (segundos) foi na ordem de 35 (cenário 1), para 137.530 (cenário 8) via PLI. Considerando o uso da meta-heurística SA, os valores nessa unidade foram de 39,07 (cenário 1) e 67,98 (cenário 8), um valor reduzido ao se comparar com a PLI. Assim, percebeu-se que o efeito tempo de processamento exerceu uma baixa influência na meta-heurística SA, ou seja, não ocorrendo um aumento exponencial conforme ocorrido na PLI, porém, apresentando uma tendência de crescimento próximo a um efeito linear. No cenário mais simples, o tempo computacional via PLI e meta-heurística SA foram muito próximos, apresentando uma vantagem à PLI por garantir o ótimo (Tabela 3). Entretanto, ao se compararem os de-

TABELA 3: Análise do tempo de processamento para os métodos de resolução nos 8 cenários.

TABLE 3: Analysis of the processing time to the resolution methods in 8 sceneries.

\begin{tabular}{lrrrrrrrr}
\hline \multirow{2}{*}{$\begin{array}{c}\text { Métodos de } \\
\text { resolução }\end{array}$} & \multicolumn{1}{c}{ Cenários } \\
\cline { 2 - 9 } & \multicolumn{1}{c}{2} & \multicolumn{1}{c}{3} & \multicolumn{1}{c}{4} & \multicolumn{1}{c}{5} & \multicolumn{1}{c}{6} & \multicolumn{1}{c}{7} & \multicolumn{1}{c}{8} \\
\hline PLI & 35 & 902 & 1.360 & 642 & 36.122 & 8.832 & 99.835 & 137.530 \\
\hdashline SA min & 38,00 & 42,00 & 46,00 & 49,00 & 54,00 & 58,00 & 63,00 & 66,00 \\
SA med & 39,07 & 43,15 & 47,10 & 50,17 & 54,76 & 59,56 & 63,90 & 67,98 \\
SA max & 40,00 & 44,00 & 48,00 & 51,00 & 56,00 & 61,00 & 65,00 & 69,00 \\
SA desvio & 0,51 & 0,53 & 0,48 & 0,51 & 0,49 & 0,55 & 0,62 & 0,56 \\
CV (\%) & 1,32 & 1,25 & 1,02 & 1,02 & 0,90 & 0,93 & 0,98 & 0,84 \\
\hline
\end{tabular}

Em que: $\mathrm{CV}(\%)$ - coeficiente de variação, desvio - desvio padrão, min - tempo mínimo obtido, med - tempo médio obtido após 100 repetições, Max - tempo máximo obtido. 
mais resultados, percebeu-se que a meta-heurística SA foi realmente um método eficiente em relação ao tempo de obtenção das soluções, confirmando as afirmativas de Suman e Kumar (2006).

$\mathrm{O}$ cenário 8 foi o que demandou maior tempo de processamento, chegando a consumir 137.530 segundos via PLI, sendo 2.023,09 vezes mais demorado que o tempo médio de processamento pela meta-heurística SA. Devido ao baixo coeficiente de variação na variável tempo de processamento, percebeu-se que a meta-heurística SA obteve um comportamento aparentemente constante para as 100 repetições, onde a variação existente pode ser atribuída a processos estocásticos presente no método. A importância de se computar o tempo de processamento está em permitir simular estratégias de planejamento da colheita florestal, onde o fator tempo pode ser uma barreira. No problema teste, foram fixadas as variáveis como taxa de juros, preço de venda da madeira e custos do empreendimento, porém, considera-se a possibilidade de variá-los ao ponto de gerar 50 cenários para um horizonte de planejamento de 14 anos (cenário 8). Logo, o tempo de processamento utilizando a PLI pode chegar a 6.876 .500 segundos ou 79,58 dias de processamento ininterrupto, já pela meta-heurística SA esse tempo seria inferior a 1 hora. A discrepância de valores mostra a importância de estudos envolvendo o desenvolvimento e aperfeiçoamento de meta-heurísticas de busca como a SA.
Falcão e Borges (2003) chegaram a conclusão, em seus estudos, que a meta-heurística SA apresentou uma melhor eficiência frente à programação linear, na resolução de problemas de grande dimensão na gestão florestal, ou seja, consumindo um menor tempo de processamento.

Apesar da vantagem no tempo de processamento, as meta-heurísticas não garantem o ótimo, e suas soluções podem ficar distantes do ótimo. $\mathrm{Na}$ Tabela 4, observa-se um resumo comparativo dessas diferenças, onde a meta-heurística SA, em todos os cenários, não se igualou com o valor do VPL obtido pela PLI, e por isso não atingiu o ótimo. Além desse fato, com o aumento da complexidade do problema, percebeu-se que a meta-heurística SA se distanciava do ótimo, passando de 4,71\% (cenário 1) para 11,40 \% (cenário 8), considerando a melhor solução obtida após 100 processamentos, para cada cenário. O uso da melhor solução como um dos critérios de comparação é referente à necessidade de se obter soluções com menor desvio do ótimo, sendo a essência de um método aproximativo.

Segundo Crowe e Nelson (2005), o tamanho do problema de agendamento da colheita florestal afeta moderadamente a habilidade da SA encontrar soluções próximas do ótimo. Um comportamento semelhante foi observado por Brumelle et al. (1998), ao utilizarem a meta-heurística busca tabu em horizontes de planejamento de 60 e 120 anos.

TABELA 4: Valores do VPL (R\$) obtido pela função objetivo nos 8 cenários.

TABLE 4: Values of the NPV (R\$) obtained by the objective function for the 8 sceneries.

\begin{tabular}{|c|c|c|c|c|c|c|c|c|c|}
\hline \multirow{2}{*}{ MET } & \multirow{2}{*}{ EST } & \multicolumn{8}{|c|}{ Cenários } \\
\hline & & 1 & 2 & 3 & 4 & 5 & 6 & 7 & 8 \\
\hline \multirow{7}{*}{$\mathrm{SA}$} & Min & 8.884 .496 & 8.670 .059 & 9.311 .969 & 9.684 .357 & 10.336 .620 & 10.468 .976 & 10.700 .700 & 10.821 .365 \\
\hline & Med & 9.669 .128 & 9.966 .542 & 10.262 .860 & 10.592 .444 & 11.160 .223 & 11.413 .381 & 11.733 .528 & 11.831 .983 \\
\hline & Max & 10.328 .954 & 10.888 .038 & 11.049 .572 & 11.372 .596 & 12.035 .942 & 12.185 .044 & 12.687 .522 & 13.067 .548 \\
\hline & Desvio & 325.504 & 398.606 & 362.739 & 375.901 & 379.267 & 401.741 & 410.909 & 460.762 \\
\hline & CV (\%) & 3,37 & 4,00 & 3,53 & 3,55 & 3,40 & 3,52 & 3,50 & 3,89 \\
\hline & $\mathrm{N}(+)$ & 99 & 81 & 41 & 9 & 70 & 1 & 42 & 24 \\
\hline & $\mathrm{N}(-)$ & 84 & 13 & 97 & 51 & 79 & 61 & 1 & 44 \\
\hline PLI & & 10.840 .050 & 11.698 .380 & 12.264 .520 & 12.801 .960 & 13.421 .320 & 13.930 .160 & 14.372 .530 & 14.748 .750 \\
\hline \multirow{2}{*}{ D } & Abs & 511.096 & 810.342 & 1.214 .948 & 1.429 .364 & 1.385 .378 & 1.745 .116 & 1.685 .008 & 1.681 .202 \\
\hline & Rel & 4,71 & 6,93 & 9,91 & 11,17 & 10,32 & 12,53 & 11,72 & 11,40 \\
\hline
\end{tabular}

Em que: MET- método de resolução; EST - estatística; Min - valor mínimo; Med - valor médio; Max - valor máximo; $\mathrm{N}(+)$ - número de processamentos para encontrar a melhor solução pela meta-heurística SA; N (-) - número de processamentos para encontrar de pior solução; D - desvio entre PLI e a meta-heurística SA; Abs - valor absoluto da diferença para o VPL entre a melhor solução obtida pela meta-heurística SA e o ótimo e Rel - porcentagem da diferença para o VPL entre a melhor solução obtida pela meta-heurística SA e o ótimo. 
A qualidade da solução obtida é influenciada por uma série de fatores, sendo um deles, e talvez o mais importante, a capacidade que a meta-heurística apresenta em escapar de ótimos locais. Sendo assim, constatou-se que o uso de meta-heurísticas na resolução de problemas de otimização não deve ser processado uma única vez, pois há riscos em obter soluções de qualidade inferior, ou seja, mais distante do ótimo. A partir da Tabela 4 observou-se que, nos 8 cenários, a obtenção das soluções com maior VPL poderia ocorrer em qualquer processamento (1 a 100), não havendo uma forma de previsão em qual processamento a mesma poderia ocorrer. Esta situação demonstra que suas soluções são independentes, e carecem de um número elevado de processamentos para a escolha da melhor solução para o problema. $\mathrm{O}$ mesmo fato foi observado na geração da pior solução por cenário. Apesar do exposto, a variação do VPL para as soluções foi considerada baixa, permanecendo na faixa de $3,37 \%$ a $4 \%$ ao longo dos 8 cenários. Crowe e Nelson (2005) comentam que a SA pode fornecer resultados eficientes ao planejamento tático no manejo florestal, no qual obtiveram de $87 \%$ a $99 \%$ de soluções próximas do ótimo, com variações nas soluções de $0,1 \%$ a $2,3 \%$. Os autores utilizaram um tempo de processamento igual a 30 minutos, 50.000 iterações e algumas variações na meta-heurística SA. Além disso, utilizaram a restrição de adjacência do tipo ARM.

Uma alternativa aos resultados obtidos foi realizada para verificar a influência do tempo de processamento na qualidade da solução obtida, ou seja, verificar o desvio em relação ao ótimo. Sendo assim, selecionou-se o cenário mais complexo (cenário 8) e se processou novamente a meta-heurística SA por 30 minutos, permanecendo com seus parâmetros iniciais constantes. Os resultados mostraram que houve uma melhora, ou seja, o desvio do VPL reduziu de 11,40 \% (30.000 iterações) para 5,16 \% (765.683 iterações), atendendo a todas as metas. Durante o processamento, notou-se que no período inicial ocorriam melhoras contínuas no valor do VPL. Contudo, as melhoras passaram a ficar escassas, ao passar de 30.000 iterações, ocorrendo a partir de grandes intervalos de tempo. Aúltima alteração na solução ocorreu por volta da iteração número 570.000, sendo interrompido o processo em 765.683 iterações, com 30 minutos de processamento.

A mesma lógica foi aplicada considerando o cenário 1 , e essa tendência se confirmou, porém, o desvio em relação ao ótimo passou de $4,71 \%$ para $4,18 \%$ com um total de $1,16 \times 10^{6}$ iterações, aproximadamente. $\mathrm{O}$ esforço computacional em tempo pode exercer uma maior influência em problemas mais complexos, porém, é necessário formular e testar esta hipótese. Uma medida para reduzir o desvio em relação ao ótimo seria trabalhar novas configurações da meta-heurística SA. Brumelle et al. (1998), trabalhando com a meta-heurística busca tabu, recorreram a estratégias de diversificar as buscas e explorar áreas não amostradas, como forma de melhorar a solução final. Rodrigues et al. (2004) estudaram os parâmetros iniciais da meta-heurística SA com o objetivo de obter a melhor configuração, e obtiveram uma solução com desvio de $5 \%$ do ótimo, porém, não utilizaram restrições de adjacência, apenas o modelo I de Johnson e Schermann (1977). Os mesmos autores constataram uma influencia dos parâmetros iniciais na meta-heurística SA.

A habilidade da meta-heurística SA em atingir as metas do problema teste é um ponto importante, pois não adianta apenas observar o valor do VPL. Dessa maneira, pôde-se constatar que as metas foram cumpridas na grande maioria, não obtendo êxito em 19 ocorrências para a restrição de adjacência do tipo URM e 15 ocorrências para as metas volumétricas, ao longo das 100 repetições/cenário. O número total de processamentos chegou a 800 (8 cenários $\mathrm{x}$ 100 repetições), no qual os detalhes das metas são expostos na Figura 4.

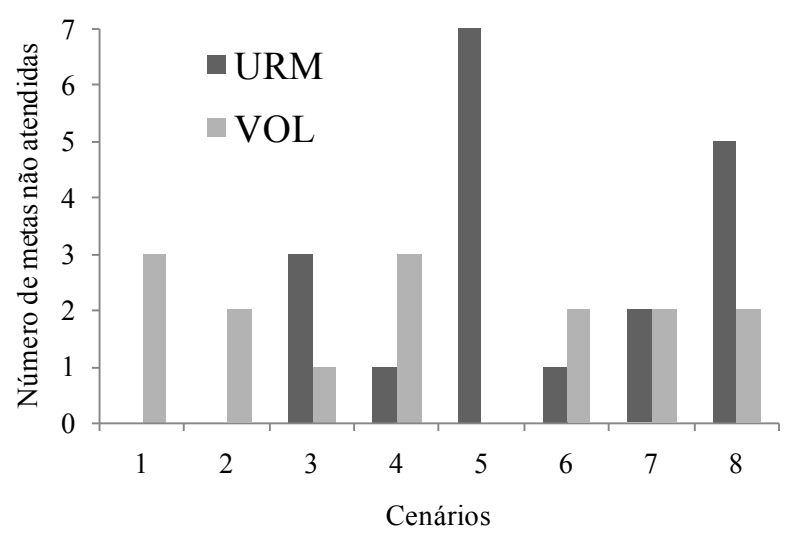

FIGURA 4: Número de metas (URM: restrição de adjacência e VOL: restrição de produção volumétrica) não atendidas pela meta-heurística simulated annealing ao longo das 100 repetições/ cenário.

FIGURE 4: Number of goals (URM: adjacency constraint e VOL: volume yield constraint) not attempted by the metaheuristic simulated annealing into 100 repetitions/scenery. 
A Figura 5 apresenta a produção volumétrica gerada para os 8 cenários, considerando a PLI e a meta-heurística SA. O desvio da produção volumétrica total obtida nos cenários ficou entre $1,69 \%$ (cenário 5 ) a 3,76 \% (cenário 3), considerando a melhor solução obtida. Sendo assim, o grau de complexidade do problema não influenciou na produção volumétrica dimensionada, conforme observado para o VPL, apresentando ainda um menor desvio de maneira geral.

Apesar dos desvios encontrados em relação ao ótimo nos 8 cenários, quando empregada a meta-heurística SA, tem-se a convicção de que a diferença encontrada não afeta o planejamento da colheita no nível tático, mesmo apresentando reduções no VPL. Essa afirmativa se baseia na realidade, pois, ao retratar um horizonte de planejamento em escala anual, tem-se sempre a presença de fatores que
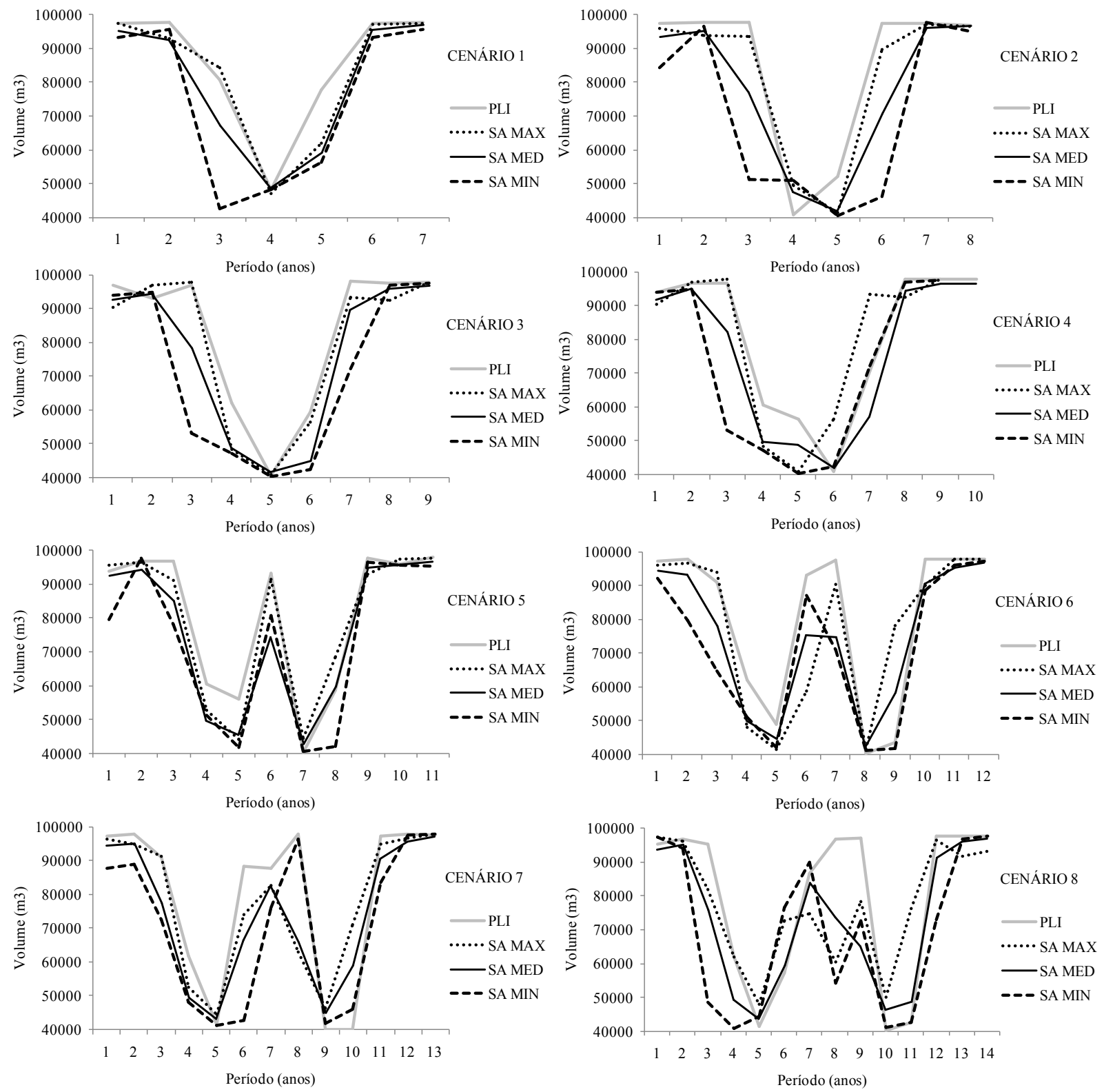

FIGURA 5: Produção volumétrica gerada nos 8 cenários considerando a PLI (Programação linear inteira) e a meta-heurística simulated annealing (Max - melhor solução, SA Med - solução média e SA Min - pior solução).

FIGURE 5: Volumetric yield produced for the 8 sceneries considering ILP (Integer linear programming) and the metaheuristic SA (Max - best solution, SA Med - medium value and SA Min - worst solution). 
influenciam o resultado final, como por exemplo, a acurácia das projeções volumétricas de crescimento e produção; a variação nos preços e custos ao longo dos anos; o ataque de pragas e doenças e danos ambientais, o que forma um cenário repleto de riscos e incertezas. Contudo, o desafio na obtenção de soluções próximas do ótimo continua, ou seja, havendo a necessidade de estudos e testes de novas meta-heurísticas, ou a sua parametrização. Além disso, segundo Crowe e Nelson (2005), em problemas demasiadamente grandes como retratados por eles e envolvendo 36.270 variáveis de decisão binária, o tempo computacional de processamento não é o ponto a explorar, pois a diferença na qualidade da solução foi pouco afetada. Os autores afirmam que a necessidade é de trabalhar estratégias na meta-heurística SA em amostrar regiões diversificadas do espaço amostral.

\section{CONCLUSÕES}

A meta-heurística SA é capaz de resolver problemas envolvendo o agendamento da colheita florestal com restrições espaciais, no qual apresenta um tempo computacional inferior ao consumido pela PLI. Entretanto, deve-se processar mais de uma vez a meta-heurística, para aumentar as chances de serem encontradas soluções próximas do ótimo, ou seja, para o estudo, com maior valor do Valor Presente Líquido. Caso contrário, há riscos de serem obtidas soluções inferiores. Uma das hipóteses a ser testada é aumentar o número de iterações do processamento, para explorar mais soluções.

A complexidade do problema não atrapalha a meta-heurística SA em obter soluções viáveis, ou seja, que atenda às metas impostas, porém, ao aumentá-la, há um maior distanciamento do ótimo.

\section{REFERÊNCIAS BIBLIOGRÁFICAS}

ALONSO, L. R. L. O problema da consideração de restrições de adjacência em um planejamento florestal. 2003. 114 f. Dissertação (Mestrado em Métodos Numéricos) - Universidade Federal do Paraná, Curitiba, 2003.

BASKENT, E. Z. Combinatorial optimization in forest ecosystem management modeling. Turkish Journal of Agriculture and Forestry, Turkey, v. 25, p. 187-194. 2001.

BELL, S.; APOSTOL, D. Designing Sustainable Forest Landscapes. Londres: Taylor \& Francis, 2008. $356 \mathrm{p}$.
BORGES, J. G.; HOGANSON, H. M. Assessing the impact of management unit design and adjacency constraints on forestwide spatial conditions and timber revenues. Canadian Journal of Forest Research, Canada, v. 29, n. 11, p. 1764-1774, 1999. BOSTON, K.; BETTINGER, P. The economic impact of green-up constraints in the southeastern United States. Forest Ecology and Management, Netherlands, v. 145, n. 3, p. 191-202, 2001.

BRUMELLE, S. et al. A tabu search algorithm for finding good forest harvest schedules satisfying green-up constraints. European Journal of Operational Research, Netherlands, v. 106, n. 2, p. 408-424, 1998.

CASTRO, R. R. Regulação de florestas equiâneas incluindo restrições de adjacência. 2007. 64 p. Dissertação (Mestrado em Engenharia Florestal) Universidade Federal de Viçosa, Viçosa, 2007.

CROWE, K. A.; NELSON, J. D. An evaluation of the simulated annealing algorithm for solving the area-restricted harvestscheduling model against optimal benchmarks. Canadian Journal of Forest Research, n. 35, p. 2500-2509, 2005.

FALCÃO, A. O.; BORGES, J. G. Heurísticas para a integração de níveis estratégico e operacional da gestão florestal em problemas de grande dimensão. Scientia Forestalis, n. 63, p. 94-102, 2003.

GLOVER, F.; KOCHENBERGER, G.A. Handbook of metaheuristics. Nova Iorque: Kluwer, 2003. $557 \mathrm{p}$.

GOMIDE, L. R.; ARCE, J. E.; SILVA, A. L. Efeito das restrições espaciais de adjacência no planejamento florestal otimizado. FLORESTA, Curitiba, v. 40, n. 3, p. 573-584, 2010.

GOYCOOLEA, M. et al. Harvest scheduling subject to maximum area restrictions: Exploring the exact approaches. Operational Research, v. 53, n. 3, p. 490-500, 2005.

HERRMAN, J.W. A history of production scheduling. p.1-22. In: HERRMAN, J.W. (Ed.) Handbook of production scheduling. Nova Iorque: Springer. 2006. 318 p.

HOOKER, J. N. A principled approach to mixed integer/linear problem formulation. p. 21. 2008. Disponível em: <(http://ba.gsia.cmu.edu/jnh/ milpmodeling.pdf)> . Acesso em: 06 agosto de 2008 . HOTVEDT, J. E. Application of linear goal programming to forest harvest scheduling. Southern Journal of Agricultural Economics, v. 15, p. 103-108, 1983.

JOHNSON, K. N.; SCHEURMANN, H. L. Techiniques for prescribing optimal timber harvest 
and investment under different objectives discussion and synthesis. Forest Science, v. 18, n. 1, p. 1-31, 1977.

KIRKPATRICK, S.; GELATT, C. D.; VECCHI, M. P. Optimization by Simulated Annealing. Science, v. 220, n. 4598, p. 671-680, 1983.

LAARHOVEN, P. J. V.; AARTS, E. H. Simulated

Annealing: Theory and Applications. Amsterdam: Springer. 1987. $204 \mathrm{p}$.

MURRAY, A. T.; GOYCOOLEA, M.; WEINTRAUB, A. Incorporating average and maximum area restrictions in harvest scheduling models. Canadian Journal of Forest Research, Canada, v. 34, p. 456-464, 2004.

MURRAY, T.; SNYDER, S. Introduction to spatial modeling in Forest managemant and natural resource planning, Forest Science, v. 46, n. 2, p. 153-154, 2000.

PEZESHK, S.; CAMP, C. V. State of the on the use of genetic algorithm in design of steel structures. p.200250. In: BURNS, S. A. (Ed.) Recent advances in optimal structural design. Reston: ASCE. 2002. $312 \mathrm{p}$.

RODRIGUES, F. L. et al. Metaheurística simulated annealing para solução de problemas de planejamento florestal com restrições de integridade. Revista Árvore, Viçosa, v. 28, n. 2, p. 247-256, 2004.

SILVA, G. F. et al. Problemas com o uso de programação linear com posterior arredondamento da solução ótima, em regulação florestal. Revista Árvore, Viçosa, v. 27, n. 5, p. 677-688, 2003.

SUMAN, B; KUMAR, P. A survey of simulated annealing as a tool for single and multiobjective optimization. Journal of the Operational Research Society, United Kingdom, v. 57, n. 10, p. 11431160, 2006.

WEINTRAUB, A. et al. Forest management models and combinatorial algorithms: analysis of state of the art. In: ANNALS OF OPERATIONS RESEARCH, 2000, San Antonio. Proceedings...San Antonio, 2000. p.271-285.

WEINTRAUB, A.; BARAHONA, F.; EPSTEIN, R. A column genetation algorithm for solving general forest planning problem with adjacency constraints. Forest Science, v. 40, n. 1, p. 142-161, 1994. ZHU, J.; BETTINGER, P.; LI, R. Additional insight into the performance of a new heuristic for solving spatially constrained forest planning problems. Silva Fennica, Finland, v. 41, n. 4, p. 687-698, 2007. 